\section{artelogie}

\section{Artelogie}

Recherche sur les arts, le patrimoine et la littérature de l'Amérique latine

$2 \mid 2012$

Mexique : espace urbain et résistances artistiques et littéraires face à la « ville générique »

\title{
Editorial - Mexique : espace urbain et résistances artistiques et littéraires face à la "ville générique "
}

\section{Christine Frérot et Pablo Avilés Flores}

\section{(2) OpenEdition \\ Journals}

Édition électronique

URL : https://journals.openedition.org/artelogie/7551

DOI : 10.4000/artelogie.7551

ISSN : 2115-6395

Éditeur

Association ESCAL

Référence électronique

Christine Frérot et Pablo Avilés Flores, «Editorial - Mexique : espace urbain et résistances artistiques et littéraires face à la « ville générique » », Artelogie [En ligne], 2 | 2012, mis en ligne le 21 janvier 2012, consulté le 07 janvier 2022. URL : http://journals.openedition.org/artelogie/7551 ; DOI : https:// doi.org/10.4000/artelogie.7551

Ce document a été généré automatiquement le 7 janvier 2022.

Association ESCAL 


\title{
Editorial - Mexique : espace urbain et résistances artistiques et littéraires face à la « ville générique »
}

\author{
Christine Frérot et Pablo Avilés Flores
}

1 Différents discours constituent le dossier thématique, de l'universitaire à l'artiste en passant par le directeur de musée, le commissaire d'exposition et l'écrivain, notre revue ayant pour ambition " par l'édition de ses numéros thématiques [...] de couvrir la plus grande variété de créations, autant intellectuelles, qu'artistiques et culturelles » en invitant leurs différents acteurs à y témoigner. Car s'il n'y a pas qu'un regard et qu'il existe des regards sur la ville, il ne fait aucun doute que les artistes, comme les écrivains, sont les témoins directs et sensibles des changements dont leurs créations sont les matériaux vivants. Nous avons donc ouvert dans le dossier thématique une section intitulée «Essai/Chronique/Témoignage» dans laquelle nous avons accueilli des textes divers qui sont ancrés dans l'espace urbain ou s'articulent avec les problématiques de la ville. La "ville générique» est donc mise en échec à chaque instant par une multiplicité de créations et de regards qui cherchent à étudier, analyser, débusquer, provoquer, composer, utiliser..., ce réservoir d'imaginaires et de symboles qu'est la capitale mexicaine, même si elle est, partiellement, globalisée.

2 L'entretien qu'a accordé en mai 2011 l'anthropologue Nestor Garcia Canclini à Christine Frérot ouvre la lecture de ce dossier thématique en nous invitant à interroger l'interculturalité et la transhistoricité de cette "ville-laboratoire» qu'est Mexico, mégalopole qui a pour capacité de faire vivre ensemble plusieurs étapes du développement historique, sans oublier, comme l'avait écrit Carlos Monsiváis, que l'Apocalypse est au coin de la rue. L'imaginaire de la ville générique conceptualisée par Rem Koolhaas a-t-il déclenché à Mexico un éveil et/ou un affrontement avec l'imaginaire nationaliste? L'article de l'architecte et historienne culturelle Johanna Lozoya s'intéresse à ce qu'elle appelle la sonore opposition entre tradition et modernité 
dans les imaginaires urbains contemporains de la mexicanité. Loin d'être conceptualisée comme une relation d'antagonisme, l'auteure considère que les deux constructions révèlent la pratique d'une construction silencieuse d'ordre historiographique: l'invention de la victimisation mexicaine moderne. Une autre anthropologue, Silvia Mancini, aborde la diversité des pratiques du culte de la Santa Muerte à Mexico dont elle analyse en profondeur l'évolution des formes et des symboliques dans une ville en mutation accélérée.

3 Les trois principaux guides touristiques qu'a choisi de commenter l'historienne d'art Christine Frérot (CITÁMBULOS Guía de asombros Ciudad de México. El transcurrir de lo insólito) ; ¿A DÓNDE VÁIS MONSIVÁIS ? Guía del DF de Carlos Monsiváis; et Nueva guía del CENTRO Histórico de México), s'inscrivent dans le développement de nouveaux discours et récits sur la ville, en dehors de la place qu'y occupe la littérature actuelle dont elle ne parlera pas ici. Différents dans la forme, le format et le fond, ils sont chacun les héritiers de cette façon plus libre de chercher une autre ville dans la ville, et de vivre sa ville. Quatre auteurs, Ana Álvarez, Fionn Petch, Valentina Rojas Loa et Christian von Wissel ont conçu le guide Citámbulos, El transcurrir de lo insólito, comme une invitation à parcourir les rues de Mexico, à dialoguer avec ses habitants et à laisser le regard subjectif construire une nouvelle cartographie des phénomènes urbains que les habitants ignoraient (et ignorent) dans leur déambulation quotidienne. En partant de la prémisse que ce sont le regard et l'imaginaire qui s'homogénéisent et non la ville, Citámbulos propose d'établir une relation plus épidermique avec Mexico pour que chaque habitant crée son propre guide de surprises, pour que l'insolite soit une évidence.

4 Le lien de la ville à la littérature est proposé dans une approche personnelle de l'écrivain et essayiste Philippe Ollé-Laprune qui s'attache aux textes de Luis Villoro et de José Emilio Pacheco, alors que Sergio Miranda Pacheco analyse en profondeur, à travers le choix de quelques poèmes, la relation entre la sensibilité poétique de Ramon Lopez Velarde et le lien avec la modernité urbaine de Mexico que l'auteur qualifie de "naufrage ». A la fois chronique et essai, le texte de l'écrivain Jaime Moreno Villareal nous entraîne, à travers une sorte de "fiction-témoignage", dans le village de San Fernando dans l'Etat de Tamaulipas, tristement célèbre pour la découverte des horreurs perpétrées par les narcos Zetas. Itala Schmelz analyse plusieurs contes de science-fiction mexicains qui abordent le thème du futur de la ville de Mexico sur un ton généralement dystopique. L'auteure s'intéresse à cette ville à partir des normes du genre de la science-fiction comme diagnostic du présent ; c'est ainsi qu'elle recherche les traces de ce futur imaginaire qui non seulement s'est déjà concrétisé mais est largement dépassé, la capitale mexicaine y étant identifiée comme l'un des centres les plus chaotiques et apothéotiques de la planète.

5 L'étroite imbrication de la ville et de la création se poursuit, avec l'art, dans la présentation de Gonzalo Ortega. Son texte n'aborde pas uniquement la création de projets d'art public dans Mexico, mais il traite aussi de la transformation radicale qui a affecté les espaces de la collectivité durant les dernières quarante années. La ville a changé, d'un environnement vierge - susceptible d'être l'objet de critiques politiques et sociales -, à sa situation actuelle, où toute proposition artistique dans la rue doit affronter la présence excessive de l'affichage publicitaire et politique. En tant qu'historienne de l'art, Elia Espinoza déclare que le « Bordo de Xochiaca » (dépotoir de la capitale) est l'installation-nature morte la plus grande que l'on puisse imaginer à 
Mexico puisqu'elle est réalisée jour après jour et indirectement par nous tous. A partir de cette prémisse conceptuelle et esthétique, elle dégage une épistémologie dans laquelle la nature-morte s'enrichit en tant que genre pictural très ancien et se déplace, pour ainsi dire, vers une installation, provoquant une sorte de correspondance et de transubstantiation des arts et des réalités visuelles-spatiales urbaines.

6 L'historienne Susana Villafuerte, en abordant le mouvement de sculpture monumentale en acier inoxydable de Tultepec (Etat de Mexico), mène une réflexion sur la création artistique contemporaine et son rapport avec les stratégies de re-création et de renouvellement des traditions d'une localité au nord de la zone métropolitaine. Dans leur texte, Colette Grandclaudon et Edgard Vidal interrogent le lien entre art et technologie en se centrant sur un détail paradoxalement délaissé, bien que très visible - un téléscripteur - d'une partie de la fresque murale de Diego Rivera dans le Palacio Nacional à Mexico. L'analyse de ce « micro-objet » les conduit inexorablement à l'observation d'un "macro-dispositif technologique" à partir duquel Diego Rivera questionne les modalités du «vivre ensemble " et d'où émerge progressivement la vision d'une mégapole moderne.

Plusieurs artistes mexicains en activité nous parlent de leurs liens anthropologiques, esthétiques et artistiques avec la ville de Mexico. Ecrit à deux mains par Marisa Lara et Arturo Guerrero, artistes multimedia qui forment le duo Siameses Company, leur texte est une réflexion sensorielle sur la ville de Mexico à partir de ses moeurs, de ses contradictions et de ses clairs-obscurs et aborde les relations complexes entre tradition et modernité. Les auteurs connaissent leur ville, ils la vivent intensément et ils en ont fait le thème de leurs peintures, sculptures, gravures, photographies, installations et performances au cours des vingt-huit ans d'un travail artistique professionnel réalisé en couple. Perla Krauze témoigne de son rapport physique à la ville. Elle réalise, au cours de ses promenades, des interventions dans les fissures qu'elle trouve au hasard dans le sol des rues, autant dans les villes que dans les zones rurales, "en comblant" ces fentes de plusieurs façons. Son objectif est de mettre en évidence ou de rendre "visible" ce qui passe inaperçu dans le quotidien du paysage urbain: la force énorme de la nature qui continue à être présente, là sur l'asphalte, et qui montre sa puissance. Le photographe Gerardo Suter propose des images imbriquées et superposées de sa vision de la capitale mexicaine qui, dans sa rétrospective de l'Antiguo Colegio de San Idelfonso à Mexico, nous offrent une sensation d'immersion, de saturation visuelle et de stimuli inattendus. Charlotte Pescayre, ethnofildefériste, a fondé le cirque Transatlancirque et elle nous explique comment ce dernier tisse des liens entre la culture mexicaine moderne de la ville de Mexico à travers le clown, la culture indigène, par la maroma zapotèque de Santa Teresa Veracruz et le son jarocho veracruzano, et la culture européenne, forte de son cirque de création.

\section{RÉSUMÉS}

Pour ce deuxième numéro d'Artelogie, nous avons modifié nos exigences éditoriales et privilégié un corpus diversifié de collaborations, étant persuadés que la thématique de la ville mexicaine 
induisait une ouverture nécessaire aux artistes-plasticiens et aux autres acteurs du monde de la culture. Nous pensons par ailleurs maintenir cette spécificité dans nos prochaines éditions. La ville de Mexico offre cette multiplicité de possibilités et d'interprétations, avec ses diverses strates imbriquées dans le temps, qu'elles soient patrimoniales, commerciales, culturelles et artistiques, rituelles et populaires. Pour l'observateur éclairé qui l'étudie et rend compte de ses analyses; pour l'artiste dont elle est l'inspiration dominante ou constitue un matériau vivant de création ; pour l'écrivain qui en fait un sujet ou un contexte de prédilection dans ses fictions..., la mégalopole mexicaine est un kaléidoscope à la fois fascinant et effrayant, séduisant et répulsif, que les articles de ce numéro vous invitent à découvrir au travers des multiples facettes de ses univers réels et symboliques.

Para este segundo número de Artelogie, dedicado a la problemática urbana en México, hemos considerado pertinente, además de contar con colaboraciones académicas, integrar testimonios y reflexiones de artistas y escritores. En efecto, la megalópolis mexicana es un caleidoscopio a la vez fascinante y espantoso, seductor y repulsivo, que los artículos de este número invitan a descubrir a través de las múltiples caras de sus universos reales y simbólicos. 